\title{
Análisis de las dimensiones del compromiso cognitivo a nivel postsecundario desde una perspectiva socio-cognitivista
}

\author{
Analysis of the cognitive engagement \\ dimensions at the post-secondary level from a
}

socio-cognitive perspective

Análise das dimensões do engajamento cognitivo no nível pós-secundário a partir de uma perspectiva sociocognitivista

ANASTASSIS KOZANITIS

\section{Resumen}

El compromiso académico está considerado como un factor importante en el proceso de aprendizaje y del éxito estudiantil. La literatura científica actual le reconoce tres componentes: conductual, afectivo y cognitivo. Los dos primeros componentes fueron ampliamente estudiados, lo que no es el caso del compromiso cognitivo. El propósito de esta investigación es de explorar la relación entre las dimensiones que constituyen el concepto de compromiso cognitivo a través de un análisis estadístico exploratorio desde una perspectiva teórica socio-cognitivista. La muestra está compuesta de 357 participantes (130 hombres y 223 mujeres) que respondieron al cuestionario del compromiso cognitivo, una versión traducida y adaptada de Student Engagement Scale. Los datos fueron sometidos a dos tipos de análisis estadístico. Primero, un análisis correlacional de tipo bivariado permitió determinar la naturaleza de los vínculos entre las dimensiones del compromiso

\footnotetext{
a Université du Québec à Montréal (LI), Montreal, Québec, Canada. Doutor em educação, e-mail: kozanitis.anastassis@uqam.ca
} 
cognitivo. Después, sun análisis univariado (ANOVA) permitió examinar el rol de ciertas variables sociodemográficas y contextuales en la predicción del compromiso cognitivo. Los resultados muestran un grado de correlación estadísticamente significativo entre los cinco constructos del compromiso cognitivo, confirmando que los mencionados antes no son independientes unos de otros. La edad de los estudiantes resulta ser la única variable sociodemográfica significativa predictiva del compromiso cognitivo.

Palabras clave: Compromiso académico. Compromiso cognitivo. Esfuerzo. Pedagogía universitaria. Motivación. Perspectiva socio-cognitiva.

\section{Abstract}

Academic engagement is considered an important factor in the learning process and student success Current scientific literature recognizes three components to engagement: behavioral, affective and cognitive. The first two components were widely studied, which is not the case of cognitive engagement. The purpose of this research is to explore the relationship between the dimensions that constitute the concept of cognitive engagement through an exploratory statistical analysis from a socio-cognitivist theoretical perspective. The sample consists of 357 participants (130 male and 223 female) who answered the cognitive engagement questionnaire, a translated and adapted version of the Student Engagement Scale. The data were subjected to two types of statistical analysis. First, a bivariate-type correlational analysis made it possible to determine the nature of the links between the dimensions of cognitive engagement. Then, an univariate analysis (ANOVA) allowed us to examine the role of certain sociodemographic and contextual variables in the prediction of cognitive commitment. The results show a statistically significant degree of correlation between the five cognitive engagement constructs, confirming that the aforementioned are not independent of each other. The age of the students turns out to be the only significant sociodemographic variable predictive of cognitive commitment.

Keywords: Academic engagement. Cognitive engagement. Effort. University pedagogy. Motivation. Socio-cognitive perspective.

\section{Resumo}

O engajamento escolar é considerado um fator importante no processo de aprendizado e no sucesso do aluno. A literatura científica atual reconhece três componentes para o engajamento: comportamental, afetivo e cognitivo. Os dois primeiros componentes foram amplamente estudados, o que não é o caso do engajamento cognitivo. O objetivo desta pesquisa é explorar a relação entre as dimensões que constituem o conceito de engajamento cognitivo por meio de uma análise estatística exploratória a partir de uma perspectiva teórica sociocognitivista. A amostra foi composta por 357 participantes (130 
do sexo masculino e 223 do sexo feminino) que responderam ao questionário de engajamento cognitivo, versão traduzida e adaptada da Student Engagement Scale. Os dados foram submetidos a dois tipos de análise estatística. Primeiro, uma análise correlacional do tipo bivariada tornou possível determinar a natureza das ligações entre as dimensões do engajamento cognitivo. Então, uma análise univariada (ANOVA) nos permitiu examinar o papel de certas variáveis sociodemográficas e contextuais na predição do engajamento cognitivo. Os resultados mostram um grau estatisticamente significativo de correlação entre os cinco construtos de engajamento cognitivo, confirmando que os acima mencionados não são independentes um do outro. A idade dos estudantes acaba por ser a única variável sociodemográfica significativa preditiva de engajamento cognitivo.

Palavras-chave: Engajamento acadêmico. Engajamento cognitivo. Esforço. Pedagogia universitária. Motivação. Perspectiva sociocognitiva.

\section{Contexto}

El tema del éxito académico en contexto postsecundario se ha vuelto une preocupación importante para muchas autoridades estatales a través el mundo. Según el Ministerio de educación de la provincia de Quebec (Canada), solo un 40\% de la población estudiantil inscrita en programas preuniversitarios y, un 37\% de aquellos inscritos en programas técnicos se gradúan dentro del tiempo prescrito (Gobierno de Quebec, 2013). Estas tasas de graduación, que están por debajo de los objetivos del gobierno, son una preocupación para la comunidad de educación superior y obligan a los investigadores a estudiar los factores que afectan el éxito, como la motivación, la perseverancia y el involucramiento académico (HAMEL; SAVARD, 2006).

Numerosos escritos en ciencias de la educación muestran que, para promover el éxito del estudiante, es importante considerar una combinación de factores, ya que esta puede influir en el desarrollo de la motivación de los estudiantes para aprender (BLUMENFELD, KEMPLER y KRAJCIK, 2006; DECI y RYAN, 2009; RADOVAN y MAKOVEC, 2015; SVINICKI y McKEACHIE, 2011; WLODKOWSKI, 2008). La motivación está considerada por Viau (2009) como el estado dinámico que predispone a un estudiante a querer involucrarse en actividades de aprendizaje para alcanzar sus metas. Es "el tipo de energía que subyace a las acciones necesarias para el éxito" (CABOT y DAGENAIS, 2016, p. 2). Este estado 
de motivación es un requisito previo para el compromiso, un tema candente en el corazón de un número creciente de investigaciones y que se discutirá en este artículo.

\section{Compromiso académico}

Desde los primeros trabajos hace al menos 30 años, tenemos una mejor comprensión del impacto del compromiso académico en el éxito. Sabemos, por ejemplo, que los estudiantes no comprometidos con el curso están menos atentos, no asisten a clases o no realizan las tareas encomendadas. Sabemos, también, que el típico estudiante no comprometido no tendrá necesariamente estos comportamientos, pero se dedicará poco al tratamiento de los contenidos (CHRISTENSON, RESCHLY y WYLIE, 2012). En ciertas disciplinas, tal estudiante completará incluso su trayectoria académica. De hecho, varios estudios demuestran que, para responder a las exigencias y salir bien librados, los estudiantes dedican mucho tiempo y esfuerzo a ejecutar rutinas y memorizar los contenidos (SMILEY y ANDERSON, 2011). Las consecuencias son, entonces, un dominio insuficiente de la materia y un bajo índice de éxito. Por el contrario, los estudiantes comprometidos son más capaces de tener un mejor rendimiento escolar, de graduarse y de hacer transiciones académicas más fácilmente (FREDRICKS, BLUMENFELD y PARIS, 2004; WILMS, FRIESEN, y MILTON, 2009).

Las investigaciones sobre el compromiso permiten también comprender mejor sus diferentes facetas a pesar de que no existe un consenso claro sobre ellas en la literatura. El compromiso es multidimensional y polisémico; un proceso interno que es difícil de observar. Sin embargo, existe un consenso sobre su importancia para el aprendizaje y sobre la influencia que los maestros tienen sobre el grado de compromiso (CHRISTENSON, RESCHLY y WYLIE, 2012). Entendido como un sistema complejo que comprende un conjunto de factores interrelacionados, internos y externos al estudiante, el compromiso tiene generalmente tres componentes: conductual, afectivo y cognitivo (AZEVEDO, 2015, BRAULT-LABBÉ y DUBÉ, 2010, FREDERICKS et al., 2004; GREENE, MILLER, CROWSON, DUKE y AKEY, 2004). 
El primero se manifiesta sobre todo por la participación del estudiante en la vida social universitaria (por ejemplo, mantener relaciones cercanas con sus compañeros) mientras que el segundo se refleja en sus intereses y valores (como guardar un vínculo entre el contenido y sus objetivos profesionales o divertirse realizando una tarea). La dimensión cognitiva es más difícil de precisar principalmente porque carece de consenso en cuanto a sus dimensiones y su medida (SINATRA, HEDDY y LOMBARDI, 2015). La mayoría de las veces se refiere a los comportamientos del alumno con respecto al saber, es decir, cómo este utiliza sus estrategias de gestión, así como las cognitivas y metacognitivas durante el aprendizaje. Es el esfuerzo y dedicación del alumno en su aprendizaje y su capacidad para pensar y usar estratégicamente lo que es pertinente para lograr el éxito deseado (TOLL, DREFS y LOCK, 2016; WILMS et al., 2009). A modo de ejemplo, un alumno cognitivamente comprometido se preocupará por aclarar conceptos, persistirá en las tareas difíciles, revisará el material aprendido y buscará fuentes de información más allá de las requeridas. Otros autores como Finn y Zimmer (2012) agregan un componente social relacionado con las reglas y normas a seguir como llegar a tiempo a clase.

Estos componentes están fuertemente influenciados por la interacción entre el individuo y el contexto de aprendizaje (FREDRICKS et al., 2004). Además, estos son sensibles a las variaciones del entorno. El principal interés por el compromiso escolar proviene de la inquietud por saber cómo crear un ambiente que tienda a activar este compromiso en todas sus dimensiones. Este es un punto importante a considerar, ya que el hecho de que un estudiante esté comprometido en sus cursos no proviene solo de una característica psicológica interna en sí mismo, sino que está modulado en gran medida por las interacciones vividas en el contexto del curso, por su percepción de la calidad de los recursos externos y personales a su alcance y por la estructura pedagógica de las situaciones de enseñanza-aprendizaje (BIGGS, 1996; FREDRICKS y McCOLSKEY, 2012). 


\section{Las dimensiones del compromiso cognitivo}

El componente cognitivo del compromiso escolar refiere a la implicación psicológica del estudiante en la construcción y comprensión de conocimientos (por cierto, este es llamado a veces compromiso psicológico) (APPLETON, CHRISTENSON, KIM y RESCHLY, 2006). Al inicio de sus trabajos, Miller, Greene y sus colegas (2015) definieron el compromiso cognitivo como las estrategias para reflexionar sobre lo que aprendemos, las reflexiones sobre las mejores formas de aprender y el esfuerzo mental para movilizar estas estrategias y reflexiones durante el aprendizaje (MILLER et al., 1996). Hoy en día, ellos lo definen de la siguiente manera: el tipo y el grado de utilización de estrategias cognitivas, la utilización de procesos de autorregulación y el grado de esfuerzo desplegado durante el estudio (GREENE, 2015). Esta definición concuerda con los resultados de diversas investigaciones sobre el aprendizaje y la motivación, incluyendo la de Fredricks y McColskey (2012) quienes mencionan que el compromiso cognitivo implica el interés y la voluntad de hacer los esfuerzos necesarios para comprender la complejidad de los contenidos y dominar habilidades difíciles.

Estamos interesados en el compromiso cognitivo a nivel del aula y de las actividades de enseñanza-aprendizaje porque le da forma, de manera directa, a las experiencias académicas del alumno y porque es, además, un requisito para el aprendizaje (SKINNER y PITZER, 2012). Entre los investigadores que estudian el compromiso cognitivo, el equipo de Miller et al. (1996) ha contribuido significativamente a desarrollar este constructo apoyándose principalmente en los marcos conceptuales de la motivación para aprender. En nuestro trabajo, utilizamos el cuestionario Cognitive Engagement Scale de Miller et al. (1996), validado muchas veces en inglés en diferentes contextos y cuya traducción en francés acabamos de validar (Leduc y Kozanitis, 2018). A continuación, describimos las dimensiones del compromiso cognitivo del "Questionnaire sur l'engagement cognitif” (QEC) las cuales son: 1) las estrategias de aprendizaje utilizadas (superficiales o profundas); 2) habilidades de autorregulación; 3) el tiempo y esfuerzo desplegados por el estudiante, y 4) la 
situación que determina el contexto en el cual los estudiantes son ubicados para aprender.

\section{Las estrategias de aprendizaje}

Inspirados por las investigaciones sobre el funcionamiento de la memoria, más específicamente por las teorías del procesamiento de la información de Craig y Lockart (1972), Miller y Greene definen el compromiso cognitivo a partir de los niveles de tratamiento de la información, ya sea de manera superficial o profunda (GREENE, 2015).

\section{Estrategias cognitivas profundas}

Basándose en las teorías cognitivas de aprendizaje, Greene et al., (2004) parten del principio de que un estudiante que utiliza diferentes tipos de estrategias para aprender intenta integrar nueva información a sus conocimientos anteriores con el fin de tener una representación mental coherente y más rica del contenido. Ellos definen así las estrategias cognitivas profundas como el uso intencional de conocimientos previos para la construcción de nuevos conocimientos a estructuras más complejas (GREENE, 2015). El aprendizaje profundo se caracteriza entonces por la utilización de funciones cognitivas de altos niveles taxonómicos. El aprendizaje profundo se correlaciona con un deseo de dominio del alumno, lo que explica en parte que un buen número de escritos hayan establecido vínculos con el éxito académico (BAETEN, KYNDT, STRUYVEN y DOCHY，2010; 2010; BIGGS，1996; DINSMORE y ALEXANDER, 2012). Cuando un alumno utiliza estrategias cognitivas profundas, este se siente personalmente absorbido por el dominio de la tarea a realizar y pone todo en práctica para comprender lo que hace. Él es consciente de sus procesos de aprendizaje en acción y los utilizará para lograr sus objetivos. De esta manera se vuelve responsable de su propia trayectoria de aprendizaje, la cual es significativa para él (CRICK, 2012). El QEC contiene 6 ítems relacionados con las estrategias profundas entre los cuales están: «Cuando estudio, comparo y distingo los 
diferentes conceptos entre ellos» $\mathrm{y}$ «Cuando aprendo nuevos conceptos, trato de pensar en aplicaciones prácticas».

\section{Estrategias cognitivas superficiales}

El aprendizaje superficial se define como el uso intencional de acciones cognitivas básicas tales como el aprendizaje memorístico o la repetición, acciones que se centran más en la repetición mecánica que en la comprensión significativa de los contenidos a aprender (GREENE, 2015). El aprendizaje superficial se correlaciona con los objetivos de rendimiento que apuntan a satisfacer las exigencias en lugar de apropiarse de la materia (BAETEN et al.; GREENE et al., 2004). Las estrategias superficiales de aprendizaje están representadas en el QEC por los siguientes ítems: «Trato de memorizar las respuestas a las preguntas de los documentos de revisión para el examen»y «Es principalmente para tener las informaciones necesarias para el examen que leo los textos del curso».

\section{La autorregulación}

Greene (2015), explica haberse inspirado en el modelo de Zimmerman y Martinez-Pons (1990) para definir los ítems relacionados con la autorregulación en sus escalas de medida. Estos permiten ver que los alumnos que se autorregulan son aquellos que se fijan objetivos de aprendizaje claros, que utilizan estrategias metacognitivas pertinentes, que movilizan múltiples métodos para estudiar eficazmente y que adaptan sistemáticamente sus esfuerzos a los resultados que obtienen para alcanzar sus objetivos (ZIMMERMAN, BONNER y KOVACH, 2000).

La autorregulación está particularmente conformada por la metacognición y la participación activa en el proceso de aprendizaje (FRÉCHETTE, 2008; ZIMMERMAN y LABUHN, 2012). Estas habilidades pueden estar enormemente influenciadas por las formas de retroacción dadas al alumno. En su revista de treinta años de investigación acerca de los modelos de autorregulación, Zimmerman y 
Labuhn (2012) señalan que las habilidades de autorregulación se desarrollan, entre otras cosas, por los procesos de retroacción establecidos en los métodos pedagógicos. La retroacción continua brindada al estudiante tiene un impacto significativo en su desarrollo, el cual tiene una influencia sobre el compromiso del alumno, lo que lo alentará a desplegar esfuerzos constantes y asiduos para alcanzar el éxito a lo largo de todos sus estudios. La autorregulación puede ser así vista como un proceso cíclico a través del cual el alumno está atento a la eficacia de sus métodos de estudio y responde a la retroacción a través de cambios resultantes de la percepción que tiene de sí mismo y de cambios manifiestos en el comportamiento. La autorregulación también implica cómo el alumno selecciona elementos de una retroacción para adaptarlos en función de sus objetivos (GREENE et al., 2004). El QEC contiene 5 ítems en relación con la autorregulación de los cuales mencionamos dos ejemplos: «Cuando termino una tarea, verifico si hay errores»; «Me aseguro de comprender bien lo que estudio».

\section{La perseverancia y el esfuerzo desplegado}

A veces llamada persistencia, la perseverancia es la dimensión que refiere principalmente al tiempo que el estudiante dedica a sus estudios hasta el logro de sus objetivos, siendo la mayoría de las veces la obtención del diploma (MOLINARI, et al. 2016). También hace referencia a la constancia con la que este estudia y a cómo lidia con los obstáculos o las limitaciones que encuentra. Kuh et al. (2006) consideran que la perseverancia es un compromiso durante un tiempo más largo y que está vinculada a las dinámicas relacionales entre el estudiante y los diferentes actores académicos. Visto desde este aspecto más macro, la perseverancia sería una consecuencia del apoyo de su entorno, de las condiciones del ambiente en el que evoluciona y de los esfuerzos que despliega para tener éxito (BOURDON et al., 2007; LAROSE et al., 2005). Miller, Greene y sus colegas (1996) integran los esfuerzos del alumno en sus estudios al constructo del compromiso cognitivo basándose particularmente en los resultados de la investigación de Dweck y Leggett (1988). Estos últimos muestran que los estudiantes que tienen metas futuras en relación con su aprendizaje muestran niveles más altos de atención y esfuerzo (GREENE, 2015). El concepto de esfuerzo es también coherente con la definición de varios autores acerca 
del compromiso cognitivo, especialmente la de Frederick y McColskey (2012) quienes consideran que la voluntad y el interés para desplegar esfuerzos a fin de integrar nociones complejas forman parte del constructo (VIAU, 2009). El QEC contiene seis ítems en relación con la perseverancia y el esfuerzo, entre ellos está: «Si tengo dificultades con una tarea asignada, persevero hasta que logre comprender».

\section{El compromiso situacional}

Las características y los parámetros del contexto en el cual tiene lugar el aprendizaje no deben pasarse por alto. Los aspectos físicos de las condiciones de enseñanza, la clase como lugar social, las formas de conducir las clases, los valores y creencias de los estudiantes con relación a los contenidos, y las diferentes relaciones humanas tienen un impacto en el compromiso cognitivo (RADOVAN y MAKOVEC, 2015). Si bien todo el entorno es determinante para el éxito académico, nosotros nos centramos en los aspectos que actúan sobre el compromiso cognitivo, es decir, aquellos que conciernen principalmente la clase y su contexto inmediato como el clima y las percepciones que los estudiantes tienen del entorno de aprendizaje (RADOVAN y MAKOVEC, 2015). Con ese fin, para estudiar el compromiso cognitivo en clase, utilizamos específicamente el trabajo de Rotgans y Schmidt (2011) quienes han agregado una dimensión situacional al compromiso académico. Esta dimensión se explica en tres aspectos interdependientes, sea cómo percibe el estudiante su compromiso durante la tarea, cómo identifica sus esfuerzos y su perseverancia mientras que realiza la tarea y de qué manera se siente absorbido por la tarea. Estos aspectos de naturaleza meta cognitiva buscan comprender el compromiso en el momento en que se produce y no solamente después de la tarea. Los trabajos de Rotgans y Schmidt (2011) son en este sentido un paso adelante para una mayor comprensión del carácter dinámico del compromiso cognitivo. Ítems como «Invierto mucho esfuerzo intelectual en este curso»y «Estoy a tal punto involucrado en este curso que me olvido de todo lo que me rodea».

El objetivo de esta investigación exploratoria es estudiar los vínculos entre las diferentes dimensiones del compromiso cognitivo y tratar de identificar qué variables 
contextuales o sociodemográficas pueden tener una incidencia sobre la implicación cognitiva. En este caso, las variables de interés seleccionadas son: la edad, el sexo y el programa de estudio (preuniversitario o técnico) de los estudiantes. Asimismo, seremos capaces de identificar si surgen diferencias entre los grupos-clases, sin tomar en cuenta las características inherentes de los mismos (tamaño del grupo, método de enseñanza, características del docente, etc.). La intención de esta investigación exploratoria es constatar si surgen tales diferencias.

\section{Metodología}

La muestra está compuesta de 357 participantes (130 hombres y 223 mujeres) que provienen de dos colegios de educación general y profesional (CEGEP) de la región Montreal, Canadá, repartidos en trece grupos-clases. De este número, 232 estudiantes están inscritos en un programa técnico, mientras que 125 estudiantes están inscritos en un programa preuniversitario. El sistema educativo de la provincia de Quebec es el único que tiene una formación postsecundaria general de dos años de duración antes de entregarse a un programa universitario. La edad promedio de los participantes es de 18 años y tres meses con una desviación estándar de dos años y tres meses. Agrupamos los participantes en dos grupos de edad, sea el grupo menores de 20 años y el de 20 años y más.

Los participantes respondieron al cuestionario del compromiso cognitivo (LEDUC y KOZANITIS, 2018), el cual es una versión traducida y adaptada de Student Engagement Scale (MILLER et al., 1996). El cuestionario consta de 33 preguntas repartidas en cinco subescalas, de las cuales cuatro provienen de la herramienta original y una quinta que ha sido agregada para tener en cuenta el compromiso situacional. La dimensión autorregulación cuenta con nueve ítems (Antes de una prueba o examen, planifico cómo estudiaré el tema); la dimensión utilización de estrategias se divide en dos categorías, sea las estrategias profundas que cuenta con siete ítems (cuando aprendo un tema nuevo, hago un resumen en mis propias palabras), y las estrategias superficiales que cuenta con cinco ítems (trato de memorizar las respuestas a las preguntas de los documentos de revisión para el 
examen); la dimensión perseverancia cuenta con ocho ítems (si tengo dificultad con una tarea, persevero hasta que logre comprender). La quinta dimensión cuenta 4 ítems y hace referencia al compromiso situacional (estoy involucrado en las tareas a realizar en este curso). Esta añadidura sigue las recomendaciones propuestas por Rotgans y Schmidt (2011) de ampliar el concepto de compromiso cognitivo a fin de considerar su carácter dinámico y poder comprenderlo cuando este se produce en una situación de aprendizaje. Los participantes responden sobre una escala tipo Likert que va de 1 (totalmente en desacuerdo) a 6 (totalmente de acuerdo). Es pertinente precisar que los estudiantes reciben la consigna de responder haciendo referencia al curso en el que se encuentran al momento de contestar. Esto con el fin de hacer comparaciones en cuanto al compromiso cognitivo según los grupos-clases. Asimismo, la herramienta incluye una pregunta para sondear la percepción de la cantidad de esfuerzo invertido en el curso en comparación con los esfuerzos normalmente invertidos en las tareas escolares. Los encuestados responden sobre una escala tipo Likert que va de a) (extremadamente limitado) a e) (extremadamente importante).

Realizamos primero análisis correlacionales de tipo bivariado a fin de determinar la naturaleza de los vínculos entre las dimensiones del compromiso cognitivo. Análisis univariados (ANOVA) han sido después realizados con el fin de examinar el rol de ciertas variables sociodemográficas y contextuales en la predicción del compromiso cognitivo.

\section{Resultados y discusión}

El cuadro 1 presenta los resultados del análisis de correlación de Pearson entre las dimensiones del compromiso cognitivo. Este análisis permite examinar la fuerza y la dirección de la relación entre las variables. El coeficiente de correlación puede tomar un valor entre - 1 y 1 , cuanto mayor es el valor absoluto del coeficiente, mayor es la fuerza de la relación entre las variables. Un valor negativo del coeficiente indica que la dirección de la relación es inversa entre las variables. 
Cuadro 1 - Inter-correlaciones bivariadas de los constructos del compromiso cognitivo mayor

\begin{tabular}{|c|c|c|c|c|c|c|}
\hline Variables & $\begin{array}{l}\text { Estrategias } \\
\text { profundas }\end{array}$ & $\begin{array}{l}\text { Estrategias } \\
\text { superfic. }\end{array}$ & $\begin{array}{c}\text { Auto- } \\
\text { regulación }\end{array}$ & Perseverancia & $\begin{array}{c}\text { Comp. } \\
\text { situacional }\end{array}$ & Esfuerzo \\
\hline $\begin{array}{l}\text { Estrat. } \\
\text { profundas }\end{array}$ & 1 & ,131* &, $642^{\star *}$ &, $327^{* *}$ &, $511^{*}$ &, $306^{* *}$ \\
\hline $\begin{array}{l}\text { Estrat. } \\
\text { superficiales }\end{array}$ & & 1 & , 114* &,$- 212^{\star \star}$ &, $122^{*}$ & , 137* \\
\hline $\begin{array}{l}\text { Autor- } \\
\text { regulación }\end{array}$ & & & 1 &, $385^{* *}$ & , 556** & ,397** \\
\hline Perseverancia & & & & 1 & , 173* &, $212^{\star \star}$ \\
\hline $\begin{array}{l}\text { Comp. } \\
\text { situacional }\end{array}$ & & & & & 1 & ,623** \\
\hline Esfuerzo & & & & & & 1 \\
\hline
\end{tabular}

* $p<0.05 ; * * p<0.001$. Fuente: Autor (2019).

Los resultados de los análisis muestran un grado de correlación estadísticamente significativo entre los cinco constructos del compromiso cognitivo, confirmando que estos constructos no son independientes unos de otros (AZEVEDO, 2015; BRAULT-LABBÉ y DUBÉ, 2010; FREDERICKS et al., 2004; GREENE et al. 2004). Estas relaciones demuestran, también, intensidades variadas, y todas, salvo una, son positivas. Las correlaciones más fuertes conciernen las estrategias profundas, la autorregulación y el compromiso situacional. Correlaciones de mediana intensidad son igualmente constatadas entre la perseverancia, las estrategias profundas y la autorregulación. Estos resultados son conformes con la conceptualización de la dimensión autorregulación, la cual reconoce la disposición y voluntad del estudiante para movilizar estrategias de adaptación con el fin de alcanzar sus objetivos (ZIMMERMAN, BONNER y KOVACH, 2000). Así pues, es interesante constatar que los estudiantes con comportamientos conscientes de autorregulación son también aquellos que hacen más usos de estrategias de aprendizaje profundas, así como de comportamientos de compromiso situacional, demostrando una participación activa en sus procesos de aprendizaje y sus deseos de invertir esfuerzos en el curso.

Las estrategias superficiales parecen tener correlaciones de débil intensidad con las estrategias profundas, la autorregulación, el compromiso situacional y la perseverancia. No obstante, si bien la intensidad de las relaciones es baja, estas son aun así significativas. Esto indica una intensión consciente en la elección de estrategias de aprendizajes y de comportamientos adaptativos según la situación de aprendizaje. 
Por otra parte, el único coeficiente de correlación de sentido negativo en el cuadro 1, indica que los estudiantes que hacen uso de estrategias superficiales tendrán menos tendencia a perseverar. Considerando el significado que se le da a la dimensión perseverancia en este estudio, este resultado puede tener implicaciones prácticas para los profesores, puesto que esta sería a la vez una consecuencia de las condiciones del entorno en el cual el estudiante evoluciona (BOURDON et al., 2007; LAROSE et al., 2005), y estaría también relacionada con la dinámica relacional entre los estudiantes y los profesores (KUH et al., 2006). De tal forma, podemos pensar que un ambiente inadecuado y una pobre relación entre los actores académicos pueden socavar, con el tiempo, la voluntad de los estudiantes para desplegar esfuerzos. O incluso, el uso de situaciones de evaluación que se basan más en la memorización puede llevar a los estudiantes a elegir estrategias de aprendizaje superficiales y, en consecuencia, a adoptar menos comportamientos de perseverancia.

Finalmente, existe una correlación positiva entre la variable esfuerzo y todas las dimensiones del compromiso cognitivo, siendo la correlación más fuerte con el compromiso situacional (0.623) y la correlación más débil con las estrategias superficiales (0.137). De manera general, este resultado muestra que los estudiantes que se sienten comprometidos cognitivamente en un curso serán también más propensos a invertir los esfuerzos necesarios para aprender el contenido del curso y tener éxito.

El cuadro 2 presenta los resultados del análisis de la varianza (ANOVA) de varios factores, permitiendo determinar qué variables pueden tener una influencia sobre el compromiso cognitivo. La estadística del test $\mathrm{F}$ permite determinar si existe una diferencia significativa entre los grupos. El valor de $p$ señala si esta estadística es significativa o no.

Cuadro 2 - Efectos de las variables sobre el compromiso cognitivo (ANOVA)

\begin{tabular}{|c|c|c|c|c|c|c|c|c|c|c|}
\hline \multirow[t]{2}{*}{ Variables } & \multicolumn{2}{|c|}{$\begin{array}{l}\text { Estrategias } \\
\text { profundas }\end{array}$} & \multicolumn{2}{|c|}{$\begin{array}{c}\text { Estrategias } \\
\text { superficiales }\end{array}$} & \multicolumn{2}{|c|}{ Autorregulación } & \multicolumn{2}{|c|}{ Perseverancia } & \multicolumn{2}{|c|}{$\begin{array}{c}\text { Compromiso } \\
\text { situacional }\end{array}$} \\
\hline & $\mathbf{F}$ & $p$ & $\mathbf{F}$ & $p$ & $\mathbf{F}$ & $p$ & $\mathbf{F}$ & $p$ & $\mathbf{F}$ & $p$ \\
\hline Edad & 5,99 & 015 & 0,06 & ,804 & 9,18 &, 003 & 13,74 &, 000 & 6,78 &, 009 \\
\hline Género & 0,19 & 819 & 1,06 & ,347 & 0,48 & ,618 & 0,73 &, 480 & 0,83 &, 436 \\
\hline Programa & 1,25 &, 265 & 4,13 &, 043 & 2,47 & , 117 & 2,68 & ,102 & 0,83 &, 436 \\
\hline Grupo-clase & 1,53 &, 102 & 1,13 & ,335 & 2,55 & ,002 & 0,56 & ,889 & 2,48 &, 003 \\
\hline
\end{tabular}

Fuente: autor (2019). 
Los resultados muestran que existe una relación significativa entre la variable edad de los estudiantes y el conjunto de constructos del compromiso cognitivo, a excepción de las estrategias superficiales, donde ninguna diferencia estadísticamente significativa ha sido observada. Asimismo, al examinar los valores del test "F" y el nivel de probabilidad de relaciones significativas, constatamos que es la variable "edad" la que explica la mayor parte de la varianza de los constructos del compromiso cognitivo, particularmente por la perseverancia $(F=13,74 \mathrm{p}<0.0001)$. Dado que son los estudiantes con más edad los que reportan un grado más elevado de compromiso cognitivo, sería oportuno para los profesores con estudiantes más jóvenes en sus clases, pensar en recurrir a prácticas pedagógicas y evaluativas que susciten este compromiso. Es interesante observar que no hay ninguna diferencia significativa para la variable género, lo que indica que hombres y mujeres presentan patrones de compromiso cognitivo similares. Igualmente, verificamos si existen diferencias entre los estudiantes matriculados en un programa técnico o preuniversitario; una vez más, ninguna diferencia estadísticamente significativa ha podido ser observada, a excepción de una pequeña diferencia en las estrategias superficiales, las que serían más utilizadas en programas preuniversitarios.

Dos hipótesis, aunque estrechamente relacionadas, podrían explicar este resultado. En primer lugar, puede ser que los estudiantes ajusten sus estrategias para responder a las exigencias de admisión de los programas universitarios con el fin de ser admitidos, y que inviertan tiempo y esfuerzo en rutinas y en memorizar los contenidos en lugar de un dominio profundo de la materia (SMILEY y ANDERSON, 2011). Además, es posible que la elección de la estrategia de aprendizaje sea consecuente con las prácticas evaluativas del maestro. Otra hipótesis probable de estos resultados puede estar relacionada con la actividad, las elecciones y la persona del profesor (CHRISTENSON, RESCHLY y WYLIE, 2012). Como se mencionó anteriormente, no controlamos estas variables en la presente investigación, lo que no significa que las metodologías de enseñanza, sean interaccionistas o más tradicionales, no pueden influir en el compromiso cognitivo de los estudiantes. Otras variables relacionadas con el estudiante, que tampoco fueron consideradas en este estudio, podrían influir en su compromiso cognitivo. Por ejemplo, podemos mencionar el 
estado de motivación, que es un requisito previo para el compromiso (CABOT y DAGENAIS, 2016), los objetivos de vida (futuros) de los alumnos, que pueden ser muy distintos (KAUFFMAN y HUSMAN, 2004), así como las metas de aprendizaje y el grado de interés por la materia (HARACKIEWICZ et al., 2007). Estas hipótesis deberán ser verificadas a través de pruebas para orientar mejor las decisiones didácticas de los docentes.

\section{Conclusión}

Mientras que numerosas investigaciones han permitido aclarar el concepto de motivación y compromiso escolar, nuestra contribución apunta a la exploración del compromiso cognitivo, un concepto que comienza a atraer la atención de investigadores y practicantes por su rol en el aprendizaje y el éxito académico en contextos postsecundarios. Una escala constituida por cinco ítems relacionados al compromiso cognitivo ha permitido recolectar datos a partir de una muestra de estudiantes del nivel colegial inscritos en programas técnicos y preuniversitarios. Los principales resultados en relación con los objetivos de esta investigación muestran relaciones significativas entre el conjunto de constructos que conforman el compromiso cognitivo. Estos muestran, también, que la edad de los estudiantes constituye una variable predictiva del compromiso cognitivo; mientras que el género de los alumnos y sus programas de estudios no presentan ninguna diferencia significativa. No obstante, algunas diferencias fueron observadas entre los gruposclases, específicamente por la autorregulación y el compromiso situacional. Futuros estudios confirmatorios deberán poder verificar el sentido y la amplitud de los vínculos que han sido establecidos por esta investigación exploratoria. De manera más precisa, los análisis ruta y los modelos de ecuaciones estructurales podrían confirmar las variables determinantes del compromiso cognitivo. Estos podrían, igualmente, considerar más variables, tales como los métodos de enseñanza, las prácticas evaluativas y los componentes que intervienen en la dinámica motivacional.

Apoyo financiero: Esta investigación recibe apoyo financiero del Consejo de Investigación de Ciencias Sociales y Humanidades de Canadá (CRSH). 


\section{Referencias}

APPLETON, J. J.; CHRISTENSON, S. L.; KIM, D.; RESCHLY, A. L. Measuring cognitive and psychological engagement: Validation of the Student Engagement Instrument. Journal of School Psychology, v. 44, n. 5, p. 427-445, 2006.

AZEVEDO, R. Defining and Measuring Engagement and Learning in Science: Conceptual, Theoretical, Methodological, and Analytical Issues. Educational Psychologist, v. 50, n. 1, 2015, p. 84-94.

BAETEN, M. et al. Using student-centred learning environments to stimulate deep approaches to learning: Factors encouraging or discouraging their effectiveness. Educational Research Review, v. 5, n. 3, p. 243-260, 2010.

BIGGS, J. Enhancing teaching through constructive alignment. Higher Education, v. 32, n. 3, p. 347364, 1996.

BLUMENFELD, P. C.; KEMPER, T. M.; KRAJCIK, J. S. Motivation and cognitive engagement in learning environments. In: KEITH, S. (Ed). The Cambridge handbook of the learning sciences. New York: Cambridge University Press, 2006.

BOURDON, S. et al. Famille, réseaux et persévérance au collégial. Phase 1: Rapport de recherche 121, 2007.

BRAULT-LABBÉ, A.; DUBÉ, L. Engagement scolaire, bien-être personnel et autodétermination chez des étudiants à l'université. Canadian Journal of Behavioural Science, v. 42, n. 2, p. 80-92, 2010.

CABOT, I.; DAGENAIS, A. Motivation scolaire. Bulletin de la documentation collégiale, 17. Montréal : Centre de documentation collégiale, 2016.

CRICK, R. D. Deep Engagement as a Complex System: Identity, Learning Power and Authentic Enquiry. In: CHRISTENSON, S. L.; RESCHLY, A. L.; WYLIE, C. Handbook or research on student engagement. Boston: Springer US, 2012. p. 675-694.

CHRISTENSON, S.; RESCHLY, A. L.; WYLIE, C. Handbook of research on student engagement. Social and Behavioral Sciences, v. 7, n. 2, p. 325-346, 2012.

DECI, E. L.; RYAN, R. M. Self-determination theory: A consideration of human motivational universals. In P. J. Corr, \& G. Matthews (Eds.), The Cambridge handbook of personality psychology. New York: Cambridge University Press, 2009. p. 441-456.

DINSMORE, D. L.; ALEXANDER, P. A. A Critical Discussion of Deep and Surface Processing: What It Means, How It Is Measured, the Role of Context, and Model Specification. Educational Psychology Review, v. 24, n. 4, p. 499-567, 2012.

DWECK, C. LEGGETT, E. A social-cognitive approach to motivation and personality. Psychological Review, v. 95, p. 256-273, 1988.

FRÉCHETTE, S. Autorégulation de l'apprentissage de l'exercice du rôle de soutien à la motivation dans le cadre de la formation des enseignants. Thèse (Doctorat inédite) - Université du Québec à Montréal, Montréal, 2008.

FREDERICKS, J. A., y W., MCCOLSKEY, W. The measurement of student engagement: A comparative analysis of various methods and student self-report instruments. In: CHRISTENSON, 
S.; RESCHLY, A.; WYLIE, K. (Eds.). The handbook of research on student engagement. New York: Springer, 2012. p. 763-741

FREDRICKS, J. A., BLUMENFELD, P. C.; PARIS, A. H. School Engagement : Potential of the Concept, State of the Evidence. American Educational Research Association, v. 74, n. 1, 59-109, 2004.

FINN, J. D.; ZIMMER, K. S. Student engagement: What is it? Why does it matter? In: S. L. Christenson, A. L. Reschly \& C. Wylie, Handbook or research on student engagement. Boston: Springer US, 2012. p. 97-131.

GOUVERNEMENT DU QUEBEC. Statistiques: Obtention d'une sanction des études collégiales. Ministère de l'enseignement supérieur, de la recherche, de la science et de la technologie, 2013.

GREENE, B. A. Measuring cognitive engagement with self report scales: Reflections from over 20 years of research. Educational Psychologists, v. 50, n. 1, p. 14-30, 2015.

GREENE, B. A., et al. Predicting high school students' cognitive engagement and achievement: Contributions of classroom perceptions and motivation. Contemporary Educational Psychology, v. 29, p. 462-482, 2004.

GREENE, B. A., y R. B., MILLER. Influence on achievement: Goals, perceived ability, and cognitive engagement. Contemporary Educational Psychology, v. 21, p. 181-192, 1996.

HAMEL, T.; SAVARD, D. Les cégeps : une grande aventure collective québécoise. Québec: Presses Université Laval, 2006.

HARACKIEWICZ, J., A. DURIK, K. BARRON, L. LINNENBRINK-GARCIA y J. TAUEUR. The role of achievement goals in the development of interest: Reciprocal relations between achievement goals, interest, and performance, Journal of Educational Psychology, v. 100, n. 1, p. 105122, 2007.

KAUFFMAN, D. F.; HUSMAN, J. Effects of time perspective on student motivation: Introduction to a special issue, Educational Psychology Review, v. 16, n. 1, p. 1-7, 2004.

KUH, G. D.; et al. What matters to student success: A review of the literature. Commissioned Report for the National Symposium on Postsecondary Student Success: Spearheading a Dialog on Student Success, 2006.

LEDUC, D.; KOZANITIS, A. L'engagement cognitif en contexte postsecondaire : traduction, adaptation et validation d'une échelle de mesure. Revue des sciences de l'éducation Mc Gill, v. 53, n. 3, p. 454-477, 2018.

LAROSE, S.; et al. Persévérance scolaire des étudiants de sciences et génie à l'université Laval: Le rôle de la culture, motivation et socialisation scientifique. Grip, Québec : Université Laval, 2005.

MILLER, R. B.; et al. Engagement in academic work: The role of learning goals, future consequences, pleasing others and perceived ability. Contemporary Educational Psychology, v. 21, p. 388422, 1996.

MOLINARI, G. et al. L'engagement et la persistance dans les dispositifs de formation en ligne: regards croisés, Distances et Médiations des Savoirs, v. 13, 2016. Disponible en:

$<$ http://journals.openedition.org/dms/1332>. Acceso en: septiembre 16, 2019. DOI:

$10.4000 /$ dms. 1332 
RADOVAN, M.; MAKOVEC, D. Adult learning environment perceptions and satisfaction in formal education: case study of four east-european countries. International education studies, v. 8, n. 2, p. 101-112, 2015.

ROTGANS, J. y H., SCHMIDT. Cognitive Engagement in the Problem-Based Learning Classroom. Advances in Health Science Education, 16, 4, 465-479, 2011.

SINATRA, G. M., B. C., HEDDY, y D., LOMBARDI. The challenges of defining and measuring student engagement in science. Educational Psychologists, v. 50, n. 1, p. 1-13, 2015.

SKINNER, E. A.; PITZER, J. R. Developmental dynamics of student engagement, coping, and everyday resilience. In: Handbook of Research on Student Engagement (p. 21-44). Boston: Springer US, 2012.

SMILEY, W., R., ANDERSON, R. Measuring Students' Cognitive Engagement on Assessment Tests: A Confirmatory Factor Analysis of the Short Form of the Cognitive Engagement Scale. Research and Practice in Assessment, v. 6, p. 17-28, 2011.

SVINICKI, M.; MCKEACHIE, J. W. McKeachie's Teaching Tips. Stategies, Research, and Theory for College and University Teachers (13th ed.). Belmont: International Edition, 2011.

TOLL, K., DREFS, M. A., \& LOCK, J. Measuring cognitive engagement. The Canadian journal for teacher research, 2016.

VIAU, R. La motivation à apprendre en milieu scolaire. Saint-Laurent : ERPI, 2009.

WILMS, J. D.; FRIESEN, S.; MILTON, P. What did you do in school today? Transforming classrooms through social, academic and intellectual engagement. (First National Report). Toronto: Canadian Education Association, 2009.

WLODKOWSKI, R. J. Enhancing adult motivation to learn: A guide to improving instruction and increasing learner achievement. San Francisco: Jossey-Bass, Inc., 2008.

ZIMMERMAN, B. J.; LABUHN, A. Theories, constructs, and critical issues. En: Self-regulation of learning: Process approaches to personal development. APA educational psychology handbook. v. 1. (pp. 399-425). Washington, DC: American Psychological Association, 2012.

ZIMMERMAN, B. J., S., BONNER, y R., KOVACH. Des apprenants autonomes : autorégulation des apprentissages. Traduction de la 1ère édition américaine par Christine Pagnoulle et Gaëtan Smets. Paris: éd. Paris De Boeck, 2000.

ZIMMERMAN, B. J., MARTINEZ-PONS, M. Student differences in self-regulated learning: Relating grade, sex, and giftedness to self-efficacy and strategy use. Journal of Educational Psychology, v. 82, p. 51-59, 1990. 